\title{
The Effect of Foot Massage on Pain and Anxiety Levels after Laparoscopic Cholecystectomy: A Randomized-Controlled Trial
}

\author{
Aysegul Abdullayev ${ }^{1}$, Ayfer Ozbas ${ }^{2}$ \\ ${ }^{1}$ Beykent University, School of Health, Istanbul, Turkey. \\ ${ }^{2}$ Istanbul University, Florence Nightingale Nursing Faculty, Istanbul, Turkey. \\ Correspondence Author: Aysegul Abdullayev \\ E-mail: aysegulabdullayev@beykent.edu.tr \\ Received: $30.01 .2021 \quad$ Accepted: 03.11.2021
}

\begin{abstract}
Objective: This study was conducted to determine the effect of foot reflexology massage performed on patients after laparoscopic cholecystectomy on pain levels, analgesic use and anxiety levels.

Methods: This prospective, experimental and randomized-controlled study was conducted with patients who underwent cholecystectomy. Ninety patients in total were divided into the experiment $(n=30)$, control $(n=30)$ and placebo $(n=30)$ groups. Foot reflexology massage was performed on the patients in the experiment group two times after surgery. Placebo massage was performed on the patients in the placebo group at the same times. Only routine treatment and care were given to the patients in the control group. The patients' pain and anxiety levels and their amounts of analgesic use within 24 hours were monitored.

Results: The patients' state anxiety scores decreased significantly from the preoperative to the postoperative measurements, and the degree of decrease did not differ between the groups $(p>0.05)$. The pain scores of the patients decreased after the first and second massage applications in all groups in comparison to their pre-massage scores, and the pain in the experiment group felt after the second massage was lower than that in the patients in the placebo group $(p<0.01)$. It was found that analgesic administration for the patients within 24 hours after the operation did not differ in terms of quantity or time of administration ( $p>0.05)$.
\end{abstract}

Conclusion: As a result, it may be stated that using reflexology with pharmacological methods to reduce postoperative pain will increase the effectiveness of pain treatment and the quality of nursing care.

Keywords: Pain, anxiety, reflexology, foot massage, cholecystectomy

\section{INTRODUCTION}

Laparoscopic cholecystectomy is the most preferred method in the treatment of gallbladder stones, one of the most common digestive system problems worldwide (1-3). After laparoscopic cholecystectomy procedures, pain may be observed due to surgical manipulation, irritation from the carbon dioxide given to the intraperitoneal area, intraabdominal pressure increase, peritonitis caused by bile and trauma caused by trocars $(4,5)$. It was reported that $13 \%$ of patients who underwent laparoscopic cholecystectomy experienced severe pain in the first week after surgery, and $17-41 \%$ were hospitalized due to pain (5). In a large-scale study with over 50,000 patients, Joshi and Kehlet evaluated pain severity levels. They demonstrated that patients experienced more severe pain in comparison to 'major' procedures after 'minor' surgical interventions such as appendectomy, tonsillectomy and cholecystectomy
(6). Additionally, the majority of patients undergoing surgical interventions experienced different levels of anxiety due to the form of anesthesia, difficulty in waking up after surgery, disability, post-operative pain, difficulty in working life, loss of body control, and fear of loss of sexual function (7-10). It is a known fact that a high anxiety level in the patient affects their pain levels in the postoperative period (11). Considering the complications of pharmacological methods such as respiratory depression, itching, nausea, vomiting and decreased gastrointestinal motility in the treatment of postoperative pain and anxiety, complementary and alternative therapy (CAT) methods have gained importance (12).

In the literature, it is stated that one of the methods of complementary and alternative medicine for relieving pain and anxiety in the postoperative period is reflexology massage 
(12-27). The International Reflexology Institute defines reflexology as a technique that is applied manually to the reflex points in the hands, feet and ears, which are associated with all glands, organs and body parts, and helps normalize body functions (28). Reflexology may often be used as a supplement to relieve the negative effects of chemotherapy and contribute to quality of life, regulate autonomic nervous system functions, strengthen the immune system and reduce stress, anxiety, agitation, tension, depression, fatigue, insomnia, as well as pain severity in acute and chronic painful conditions $(22,29-34)$.

However, there is a lack of studies in the literature that clearly demonstrate the effects of placebo-controlled reflexology massage. This study was planned and performed to determine the effect of foot reflexology massage on pain and anxiety levels in patients undergoing laparoscopic cholecystectomy surgery.

\section{METHODS}

\subsection{Study Design and Setting}

The research was conducted in a general surgery clinic of a university hospital in Istanbul between September 2017 and November 2018 with a prospective, single-center, experimental and randomized-controlled design. The study was started after receiving the approval of the clinical research ethics committee of the hospital (Date: 20.03.2017, Decision No: 001). Before starting the research process, clinical nurses were informed about the research. Patients who met the research criteria were interviewed and informed about the research, their questions were answered, and their consent was obtained verbally and in writing.

\subsection{Participants}

The population of the study included all patients undergoing laparoscopic cholecystectomy surgery within the specified time interval. Power analysis was performed using the G*Power 3.1 program to determine the minimum number of participants, and the power of the test was calculated. For the power of the study to exceed $80 \%$, it was calculated that a total of 84 people, including 28 people per group, should be reached with a $5 \%$ significance level and a 0.25 effect size $(t$ $=1.404$; effect size $d=0.25$ ). Considering that there could be losses during the research period, it was decided to admit a total of 90 patients, including 30 in each group.

The randomization of the patients who met the sampling criteria was performed using the closed envelope method, and the patients were randomly divided into three groups as foot reflexology massage (experiment group), placebo foot massage (placebo group) and no intervention (control group).

Patients without any health problems related to the foot (local infection, open lesion/wound, scar tissue, edema), who were aged 18 or older, without communication difficulties and mental deficiencies, without any complications (e.g., bleeding, nausea, vomiting, thrombophlebitis) after surgery, without the use of any medication such as analgesics before the first massage planned to be performed after surgery, without patient-controlled analgesia after surgery, with no drain placed in the surgical site, without addiction disorders and with no chronic pain in any part of their body were included in the study. The randomization of the study is shown in CONSORT (Consolidated Standards of Reporting Trials) 2010 (Figure 1)

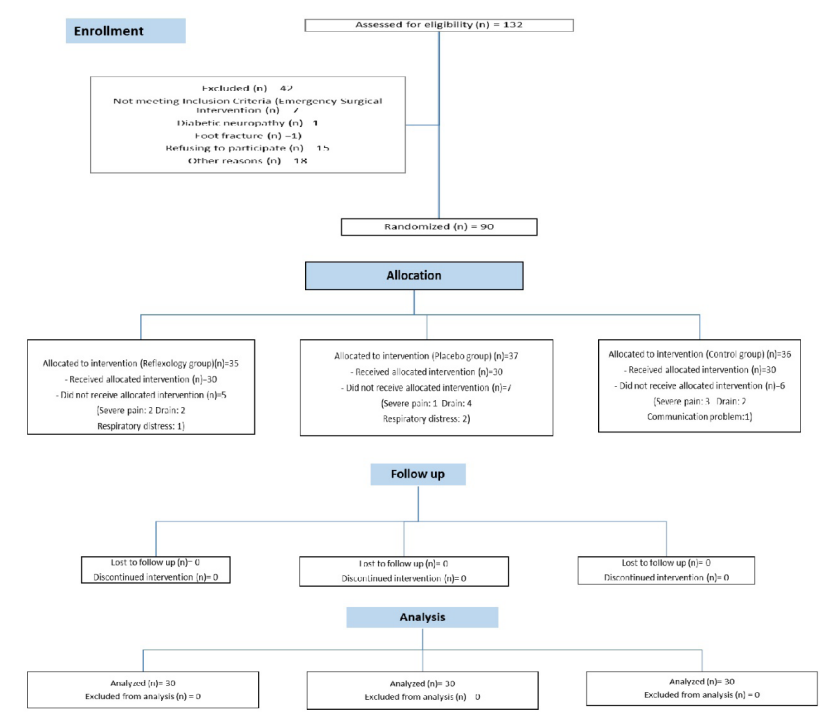

Figure 1: CONSORT flow diagram

\subsection{Intervention}

After the patients were admitted to the ward on the day of their surgical operation, a data collection form containing information about the patient's personal and medical history was completed. The content and method of the study, the scales to be used in the evaluation of the variables and the ethical principles that would be followed during their participation in the study were introduced to the patient. The vital signs of the patient (blood pressure, pulse rate, respiratory rate, oxygen saturation) were measured and recorded.

First Stage: In the early postoperative period, when the patient's location, time and person orientation was achieved, the severity and quality of the pain was evaluated. Their vital signs were measured. Foot massage was performed for the experiment group and placebo group based on their protocols specified above. Pain intensity and vital signs were re-evaluated 10 minutes after the end of the massage. The measurements were repeated at the same time as the other group, and no intervention was performed for the control group.

The verbal expression of the patient was excluded from the scope of the study by administering analgesics at the request of a physician to the patients whose pain severity was unbearable based on the observation of the researcher or the patient's physiological symptoms. From among a total of 132 patients who received laparoscopic cholecystectomy operations throughout 
the study period, the study excluded 7 patients as they received emergency surgical interventions, 15 as they did not provide consent to participate in the study, 3 as they experienced respiratory distress after the surgery, 8 as drains were placed into them after the surgery, 5 as they experienced severe pain after the surgery, 3 as it was not possible to communicate with them in the postoperative period, one patient as they had diabetic neuropathy and another as they experienced a fracture in their foot in a recent time.

Second Stage: When the half-life of the analgesics given to the patients in the postoperative recovery unit at the 3rd hour after the surgery had expired, the patients in the experiment and placebo groups received a second foot massage. Pain levels and vital signs were measured in the same way as in the previous stage.

Third Stage: At the 4th hour after surgery, the State Anxiety Inventory was re-applied to all patients. Analgesics and their usage amounts were recorded within 24 hours after surgery.

\section{Experiment group Foot Reflexology Protocol}

The patients were informed about the treatment protocol. At the beginning of the massage, foot warming movements were performed for about 5 minutes. Light pressure was applied to the solar plexus area of the feet for one minute. First, reflexology was applied to the gallbladder and digestive and musculoskeletal areas of the right foot.

Reflexology massage was performed only on the areas related to the digestive system and the musculoskeletal system because there is no area belonging to the gallbladder in the left foot. Light pressure was applied to the solar plexus area of the feet for one minute, and the treatment was terminated. The reflexology massage to the right and left feet was administered over three periods, lasting 30-35 minutes in total.

\section{Placebo Group Massage Protocol}

The protocol was explained to the patients. Foot heating with light pressure was performed only on the dorsal areas of the patients in this group because the plantar region of the foot is associated with the gallbladder and other reflexology regions of the digestive system. The massage applied on the right and left feet was performed for a total of 10-15 minutes.

The reflexology massage was performed by the researcher who has been trained and certified in this field under the supervision of a physician. The massage protocols that were determined for the experiment and placebo groups were evaluated by two reflexologists who are experts in their field. The vital signs of the patients were monitored five times using a clinical blood pressure measurement device and a pulse oximeter. The specified measurement times were as follows: (Z1) before surgery, (Z2) before 1st massage, (Z3) 10th min after 1st massage, (Z4) before 2 nd massage, and (Z5) 10th min after 2nd massage.

\subsection{Data Analysis}

The data obtained in the study were analyzed using the Statistical Package for the Social Sciences 2018 program. Whether or not the data met assumptions of normal distribution was tested, frequencies, percentages and means were used as descriptive statistics, and Chi-squared test, t-test, one-way analysis of variance (ANOVA), regression and correlation analyses or MannWhitney $U$ and Kruskal-Wallis tests were used accordingly based on the normality of data distribution.

\section{RESULTS}

It was determined that each group had similar introductory characteristics in their first measurements (Table 1). In the measurements before the surgery, the mean trait anxiety scores of the experiment group, control group and placebo group were $37.63 \pm 6.30,41.40 \pm 7.98$ and $36.26 \pm 6.37$, respectively. There was no statistically significant difference between the trait anxiety scores of the groups $(p>0.05)$. Similarly, in the intergroup comparisons, there was no difference between the mean preoperative state anxiety scores of the patients in the groups (experiment: $36.76 \pm$ 10.75; control: $38.40 \pm 8.90$; placebo; $39.83 \pm 10.66$ ) or their mean postoperative state anxiety scores (experiment: $29.10 \pm 6.67$; control: $31.76 \pm 8.35$; placebo: $31.16 \pm 6.94$ ) ( $p>0.05)$. In the intragroup comparisons, it was found that the mean postoperative anxiety scores in all groups were significantly lower in comparison to their mean preoperative scores $(p<0.05)$, but there was no statistically significant intergroup difference among the groups in terms of their mean postoperative scores $(p<0.05)$ (Table 2$)$.

The mean pain scores of the patients in all groups after the 1st massage were significantly lower than their mean scores before the 1 st massage $(p<0.05)$. Additionally, the mean pain scores expressed by the patients in all groups before the 2 nd massage were lower than those they expressed before the 1st massage, and the mean pain scores expressed after the 2nd massage were lower than those before the 2 nd massage $(p<0.05)$ (Table 3$)$. According to the results of the intergroup comparisons of the mean pain scores of the patients before and after the first and second massages, the patients in the experiment group had lower pain scores after the 2nd massage than those in the placebo group $(p<0.01)$. However, it was not possible to precisely reveal between which groups the pain score after the 1st massage significantly differed in the statistical sense $(p=0.01)$ (Bonferroni test) (Table 4).

When the amounts of analgesics administered to the patients in the experiment, control and placebo groups in the first 24 hours after the operation were examined according to the drug types, it was determined that, based on the mean values, $18 \mathrm{mg}$ tenoxicam, $2200 \mathrm{mg}$ paracetamol and $35.33 \mathrm{mg}$ tramadol were administered to the patients in the experiment group; $16 \mathrm{mg}$ tenoxicam, $1666.80 \mathrm{mg}$ paracetamol and $65.84 \mathrm{mg}$ tramadol were administered to the patients in the control group, and $18.14 \mathrm{mg}$ tenoxicam, $1166.58 \mathrm{mg}$ paracetamol and $54.66 \mathrm{mg}$ tramadol were administered in the placebo group. There was no statistically significant difference between the groups in terms of the amounts of analgesics given to the patients within the first 24 hours after the operation ( $p>0.05)$. 
Table 1. Introductory characteristics of patients $(N=90)$

\begin{tabular}{|c|c|c|c|c|}
\hline \multicolumn{2}{|c|}{ Introductory Characteristics } & \multirow{2}{*}{$\begin{array}{c}\text { Experiment }(n=30) \\
\text { Mean } \pm S D\end{array}$} & \multirow{2}{*}{$\begin{array}{c}\text { Control }(n=30) \\
\text { Mean } \pm \text { SD }\end{array}$} & \multirow{2}{*}{$\begin{array}{c}\text { Placebo }(n=30) \\
\text { Mean } \pm \text { SD }\end{array}$} \\
\hline & & & & \\
\hline \multirow[t]{2}{*}{ Age } & & $46.93 \pm 14.74$ & $47.06 \pm 11.50$ & $42.06 \pm 11.67$ \\
\hline & & $n(\%)$ & $\mathrm{n}(\%)$ & $\mathrm{n}(\%)$ \\
\hline \multirow[t]{2}{*}{ Sex } & Female & $16(53)$ & $20(67)$ & $23(76)$ \\
\hline & Male & $14(47)$ & $10(33)$ & $7(24)$ \\
\hline \multirow[t]{5}{*}{ Education Level } & Illiterate & $0(0)$ & $0(0)$ & $1(3)$ \\
\hline & Primary school & $4(13)$ & $6(20)$ & $2(7)$ \\
\hline & Secondary school & $1(3)$ & $0(0)$ & $2(7)$ \\
\hline & High school & $2(7)$ & $11(37)$ & $8(27)$ \\
\hline & Undergraduate and Graduate & $23(77)$ & $13(43)$ & $17(57)$ \\
\hline \multirow[t]{2}{*}{ Marital Status } & Married & $26(87)$ & $26(87)$ & $21(70)$ \\
\hline & Single & $4(13)$ & $4(13)$ & $9(30)$ \\
\hline \multirow[t]{5}{*}{ Job } & Housewife & $9(30)$ & $13(43)$ & $9(30)$ \\
\hline & Student & $0(0)$ & $1(3)$ & $0(0)$ \\
\hline & Civil Servant & $7(23)$ & $5(17)$ & $8(27)$ \\
\hline & Self-Employed & $8(27)$ & $8(27)$ & $9(30)$ \\
\hline & Retired & $6(20)$ & $3(10)$ & $4(13)$ \\
\hline \multirow[t]{3}{*}{ Monthly Income } & Income less than expenses & $3(10)$ & $3(10)$ & $2(7)$ \\
\hline & Income equal to expenses & $16(53)$ & $20(67)$ & $22(73)$ \\
\hline & Income more than expenses & $11(37)$ & $7(23)$ & $6(20)$ \\
\hline \multirow[t]{3}{*}{ Social Security } & SSI & $0(0)$ & $4(13)$ & $3(10)$ \\
\hline & Private Insurance & $29(97)$ & $26(87)$ & $26(87)$ \\
\hline & No Social Security & $1(3)$ & $0(0)$ & $1(3)$ \\
\hline \multirow[t]{2}{*}{ Place of Residence } & City & $29(97)$ & $30(100)$ & $30(100)$ \\
\hline & District & $1(3)$ & $0(0)$ & $0(0)$ \\
\hline \multirow[t]{2}{*}{ Smoking } & Yes & $7(23)$ & $9(30)$ & $13(43)$ \\
\hline & No & $23(77)$ & $21(70)$ & $17(57)$ \\
\hline \multirow[t]{2}{*}{ Alcohol Use } & Yes & $4(13)$ & $2(7)$ & $3(10)$ \\
\hline & No & $26(87)$ & $28(93)$ & $27(90)$ \\
\hline
\end{tabular}

Table 2. Comparison of the state and trait anxiety scale scores before and after surgery $(N=90)$

\begin{tabular}{|c|c|c|c|c|c|c|c|c|}
\hline State and Trait Anxiety Scale & \multirow{2}{*}{\multicolumn{3}{|c|}{$\begin{array}{c}\text { Experiment }(n=30) \\
\text { Mean } \pm S D\end{array}$}} & \multicolumn{2}{|c|}{ Control $(n=30)$} & Placebo $(n=30)$ & \multirow{2}{*}{$x^{2}$} & \multirow{2}{*}{$\mathbf{P}$} \\
\hline Scores & & & & \multicolumn{2}{|c|}{ Mean \pm SD } & Mean \pm SD & & \\
\hline Trait Anxiety Before Surgery & \multicolumn{3}{|c|}{$\frac{\text { IVlean I SD }}{3763+630}$} & \multicolumn{2}{|c|}{$41.40 \pm 7.98$} & $36.26 \pm 6.37$ & 3.127 & 0.20 \\
\hline Preoperative State Anxiety (D1) & \multicolumn{3}{|c|}{$36.76 \pm 10.75$} & \multicolumn{2}{|c|}{$38.40 \pm 8.90$} & $39.83 \pm 10.66$ & 2.009 & 0.36 \\
\hline Postoperative State Anxiety (D2) & \multicolumn{3}{|c|}{$29.10 \pm 6.67$} & \multicolumn{2}{|c|}{$31.76 \pm 8.35$} & $31.16 \pm 694$ & 1.930 & 0.38 \\
\hline Multiple Comparison & Z & $\mathbf{P}$ & Z & $\mathbf{P}$ & Z & \begin{tabular}{l|l}
$\mathbf{P}$ & \\
\end{tabular} & & \\
\hline$D 1-D 2$ & -4.155 & $<0.001^{*}$ & -4.112 & $<0.001^{*}$ & -4.417 & $<0.001^{*}$ & & \\
\hline
\end{tabular}

${ }^{*} p<0.05$ (Wilcoxon test, Kruskal-Wallis test)

Table 3. Comparison of patients' mean pain scores before and after foot massage-1 $(N=90)$

\begin{tabular}{|c|c|c|c|c|c|c|c|c|}
\hline \multirow{2}{*}{ Pain Monitoring Times } & \multicolumn{2}{|c|}{ Experiment $(n=30)$} & \multicolumn{2}{|c|}{ Control $(n=30)$} & \multicolumn{2}{|c|}{ Placebo $(n=30)$} & $x^{2}$ & $\mathbf{P}$ \\
\hline & \multicolumn{2}{|c|}{ Mean \pm SD } & \multicolumn{2}{|c|}{ Mean \pm SD } & \multicolumn{2}{|c|}{ Mean \pm SD } & & \\
\hline 1. Before reflexology massage (A1) & \multicolumn{2}{|c|}{$7.46 \pm 1.87$} & \multicolumn{2}{|c|}{$6.70 \pm 2.49$} & \multicolumn{2}{|c|}{$7.46 \pm 1.96$} & 1.984 & 0.371 \\
\hline 1. After reflexology massage 10th $\min (\mathrm{A} 2)$ & \multicolumn{2}{|c|}{$5.23 \pm 2.22$} & \multicolumn{2}{|c|}{$5.70 \pm 2.79$} & \multicolumn{2}{|c|}{$6.83 \pm 2.26$} & 6.178 & $0.04 *$ \\
\hline 2. Before reflexology massage (A3) & \multicolumn{2}{|c|}{$4.60 \pm 2.02$} & \multicolumn{2}{|c|}{$3.80 \pm 2.85$} & \multicolumn{2}{|c|}{$5.13 \pm 2.45$} & 5.263 & 0.07 \\
\hline 2. After reflexology massage $10^{\text {th }} \min (\mathrm{A} 4)$ & \multicolumn{2}{|c|}{$2.70 \pm 2.15$} & \multicolumn{2}{|c|}{$3.16 \pm 2.19$} & \multicolumn{2}{|c|}{$4.36 \pm 2.44$} & 7.184 & $0.02 *$ \\
\hline Multiple Comparison & $\mathrm{Z}$ & $\mathbf{P}$ & $\mathrm{Z}$ & $p$ & $\mathrm{z}$ & $\mathbf{P}$ & & \\
\hline$A 1-A 2$ & -4.575 & $<0.001 *$ & -2.956 & $<0.001^{*}$ & -2.724 & $<0.001 *$ & & \\
\hline$A 1-A 3$ & -4.302 & $<0.001 *$ & -4.301 & $<0.001^{*}$ & -4.292 & $<0.001 *$ & & \\
\hline A3-A4 & -4.529 & $<0.001^{*}$ & -1.978 & $0.04 *$ & -3.630 & $<0.001 *$ & & \\
\hline
\end{tabular}

${ }^{*} p<0.05$ (Wilcoxon test, Kruskal-Wallis test) 
Table 4. Comparison of patients' mean pain scores before and after foot massage $-2(N=90)$

\begin{tabular}{|c|c|c|c|c|c|c|}
\hline & Groups & $\mathbf{n}$ & $\begin{array}{l}\text { Mean } \\
\text { Rank }\end{array}$ & $\mathbf{U}$ & Z & $\mathbf{P}$ \\
\hline \multirow{3}{*}{$\begin{array}{l}\text { 1. (A2) Pain } \\
\text { Score after } \\
\text { Reflexology } \\
\text { Massage }\end{array}$} & $\begin{array}{l}\text { Experiment } \\
\text { Placebo }\end{array}$ & $\begin{array}{l}30 \\
30 \\
\end{array}$ & $\begin{array}{l}24.82 \\
36.18 \\
\end{array}$ & 279.50 & -2.547 & 0.01 \\
\hline & $\begin{array}{l}\text { Experiment } \\
\text { Control }\end{array}$ & $\begin{array}{l}30 \\
30\end{array}$ & $\begin{array}{l}28.67 \\
32.33\end{array}$ & 419.50 & -0.455 & 0.64 \\
\hline & $\begin{array}{l}\text { Placebo } \\
\text { Control } \\
\end{array}$ & $\begin{array}{l}30 \\
30 \\
\end{array}$ & $\begin{array}{l}34.12 \\
26.88 \\
\end{array}$ & 341.50 & -1.620 & 0.10 \\
\hline \multirow{3}{*}{$\begin{array}{l}\text { 2. (A2) Pain } \\
\text { Score after } \\
\text { Reflexology } \\
\text { Massage }\end{array}$} & $\begin{array}{l}\text { Experiment } \\
\text { Placebo } \\
\end{array}$ & $\begin{array}{l}30 \\
30 \\
\end{array}$ & $\begin{array}{l}24.73 \\
36.27 \\
\end{array}$ & 277.00 & -2.579 & $0.009 *$ \\
\hline & $\begin{array}{l}\text { Experiment } \\
\text { Control }\end{array}$ & $\begin{array}{l}30 \\
30\end{array}$ & $\begin{array}{l}28.67 \\
32.33\end{array}$ & 395.00 & -0.822 & 0.41 \\
\hline & $\begin{array}{l}\text { Placebo } \\
\text { Control }\end{array}$ & $\begin{array}{l}30 \\
30 \\
\end{array}$ & $\begin{array}{l}34.62 \\
26.38 \\
\end{array}$ & 326.50 & -1.843 & 0.06 \\
\hline
\end{tabular}

$\left({ }^{*} p<0.01\right)$ (Bonferroni test)

\section{DISCUSSION}

In the literature, it has been reported that most patients who undergo surgical interventions experience different degrees of anxiety and increased perioperative anxiety levels raise postoperative morbidity and mortality rates, delay wound healing and extend hospital stay, as well as increasing postoperative pain levels and requirement of analgesics (711).

In the study by Hudson et al. on the effects of hand reflexology massage performed on patients undergoing minimally invasive varicose vein surgery on their pain and anxiety, it was found that the levels of the anxiety felt by the patients during the procedure in the treatment group were significantly lower than those in the control group (25).

Koraş and Karabulut (2018) examined the effects of foot massage performed on patients undergoing laparoscopic cholecystectomy on their pain and anxiety levels, and the postoperative state anxiety score of the experiment group was found to be lower than that in the control group (12). In a study with 60 patients $(36.7 \%$ of whom underwent cholecystectomy) who had undergone abdominal surgery, similarly, there was no difference between the basal anxiety levels of the experiment and control groups, and the anxiety level was lower in the foot massage group after the third application (35). In another study conducted with patients who had undergone digestive system surgery, it was stated that there was no difference between the baseline anxiety levels of the experiment and control groups, as well as finding a decrease in the anxiety levels of the experiment group after the intervention (19). Besides, the decrease in the anxiety levels of the experiment group after the implementation was significantly higher (19).

In many studies examining the effect of foot massage on different levels of anxiety after surgery with different protocols in different patient groups such as abdominal surgery, tibial fracture, total abdominal hysterectomy, coronary artery bypass grafting and coronary angiography, it has been reported that the anxiety levels of the patient groups that received foot massage were significantly lower than those in the control groups $(17,22,25,36-38)$.

In this study, the mean postoperative state anxiety scores of the patients in the experiment, control and placebo groups decreased significantly in comparison to their mean preoperative scores $(p<0.05)$. In the intragroup comparisons, no significant difference was found between the mean preoperative and postoperative state anxiety scores in any of the groups $(p>0.05)$. Although there was a decrease in the levels of postoperative anxiety in all three groups, the finding the difference between the groups in terms of this drop was not significant was interpreted to be dependent on factors such as the duration of the massage, application areas, application technique and application amount.

The effective treatment of postoperative pain in surgical patients contributes to the psychologic state of the patient, increases their quality of life and comfort and prevents the negative effects of pain on body systems (13). In a study examining the effects of ear acupuncture and foot reflexology massage on patients undergoing appendectomy, it was revealed that, although the pain levels in all groups decreased after receiving the acupuncture intervention, the decrease was greater in the patients in the experiment group (39). In the study by Shehata et al. (2016), the effect of foot massage performed on patients undergoing abdominal surgery in two sessions on these patients' pain levels was examined, and after the first and second applications, the expression of the pain by the patients in the experiment group was found to be lower than that of the patients in the control group (26). In the study by Çankaya and Saritaş on patients undergoing laparoscopic cholecystectomy, it was revealed that the pain level in the experiment group was lower than that in the control group as a result of conventional foot massage performed for 10 minutes (16). Although there was no significant difference between the pain levels of the experiment and control groups before the massage intervention in the studies by Youssef and Hassan (2017) $(\mathrm{N}=60)$ and Chanif (2012) $(\mathrm{N}=40)$ among patients who underwent abdominal surgery, it was reported that the degree of pain reduction was significantly greater than that in the control group $(35,36)$.

Similar results have been achieved in the literature after foot massage in different patient groups such as breast surgery, cesarean surgery, tibia fracture surgery, CABG surgery and total abdominal hysterectomy patients $(14,17-19,22,23)$.

Unlike these studies, Koraş's study with patients who underwent cholecystectomy revealed no statistically significant difference between the mean pain intensity scores at the 5th and 30th minutes after massage in the experiment and control groups. Additionally, when the mean pain intensity scores of the patients at the 60th, 90th and 120th minutes were compared, the mean scores of the experiment group were found to be lower than those of the control group (12). In the study by Hudson et al. (2015), the effect of hand reflexology massage performed on patients 
undergoing minimally invasive varicose vein surgery during the procedure on pain was examined, it was determined that the pain intensity scores of the experiment and control groups were not significantly different, and the duration of the pain in the experiment group was shorter (25). In the study conducted by Silverdale and Roodhouse (2019) with 38 patients who underwent radical cystectomy due to bladder cancer, the patients were divided into 3 groups as the wholebody massage group, foot massage group and a group where both massages were performed together, and the protocols were performed in two sessions. It was revealed that pain levels after both treatments were lower in all groups than before the massage, but the degree of reduction did not differ between the groups (40).

In this study, although the mean pain scores of the patients in all groups decreased over time, the pain felt in the experiment group after the second massage was found to be significantly lower than that in the placebo group $(p<0.01)$. However, it was not possible to precisely determine between which groups the pain score after the 1st massage differed significantly $(p=0.01$ ) (Table $4-15)$. In the study, the decrease in the mean pain scores in all patient groups in the postoperative period in comparison to their preoperative scores was consistent with the literature; however, the fact that the decrease in the mean pain scores was not significantly different between the experiment group and the other groups differed from the literature. Koraş and Karabulut (2018) identified no significant difference between the pain levels of the experiment and control groups at the 5th and 30th minutes after foot massage. This difference might have been due to the long-term evaluation of the effects of massage, similar to that of Koraş and Karabulut (12).

Analgesics, which form the basis of pain therapy, are drugs that are used to control pain in acute and chronic pain syndromes (41). In spite of the new knowledge and experience in this field, complementary and alternative treatment methods such as reflexology have become important in the management of post-operative pain and anxiety, considering that pharmacological methods may cause complications such as respiratory depression, nausea, vomiting, itching, convulsions and decreased gastrointestinal motility (12).

In a study in which the effect of foot reflexology massage performed with ear acupuncture on the use of analgesics in patients undergoing appendectomy was analyzed, there was no difference between the analgesic use amounts of the patients in the placebo and control groups, whereas the amount of analgesic use in the experiment group was lower (39). In a study performed with a 3rd session of foot reflexology massage after cancer surgery of the digestive system, it was revealed that the patients in the experiment group used smaller quantities of opioid analgesics than those in the control group (19). In a study conducted using similar methods in patients undergoing cholecystectomy, the quantities of analgesics required in the patients in the experiment and control groups were compared, and it was revealed that the control group used analgesics at higher quantities than the experiment group (12). Similar results were obtained in Asadisker's (2011) CABG group and Öztürk et al.'s (2017) study in patients undergoing abdominal hysterectomy $(14,22)$.

In this study, when the quantities of the analgesics given to the patients in the experiment, control and placebo groups within the first 24 hours after the operation were examined based on the types of drugs, it was observed that all patients were treated with one or more types of medications such as tenoxicam, paracetamol and tramadol after surgery. It was observed that there was no significant difference in the quantities of the analgesics given to the patients within the first 24 hours after the operation between the groups, and accordingly, these results were not similar to those reported in the literature $(p>0.05)$. It is thought that this incompatibility between this study and others in the literature may have been due to the use of pharmacological pain relief methods rather than nonpharmacological pain relief methods in postoperative care for various reasons (e.g., the crowdedness of the hospital, the excess workload of the nurses) in the hospital where the study was conducted.

\section{CONCLUSION}

As a result of this research, it was found that foot reflexology massage performed in the postoperative period did not have a significant effect on the mean state anxiety scores and physiological parameters of the patients. Additionally, the patients' pain scores after the first and second massage decreased in all groups in comparison to their scores before the massage, and the severity of the pain that the patients in the experiment group reported after the second massage was lower than that reported by the patients in the placebo group. Consequently, it may be stated that using reflexology with pharmacological methods to reduce postoperative pain will increase the effectiveness of pain treatment and the quality of nursing care.

Acknowledgements: We sincerely thank all of the participants in the present study

\section{REFERENCES}

[1] Lammert F, Gurusamy K, Ko CW. Gallstones. Nature Reviews / Disease Primers 2016; (2):1-17

[2] Oddsdottir M, Pham TH, Hunter JG. Gallbladder and the extrahepatic biliary system. In: Bruncardi FC (Ed.) Schwartz's Principles of Surgery. 9. Edition Güneş Medical Bookstore; 2010 p.1136- 1164.

[3] Tazuma S, Unno M, Igarashi Y, Inui K, Uchiyama K, Kai M. Evidence-based clinical practice guidelines for cholelithiasis. Journal of Gastroenterology 2019;(52): 276-300.

[4] Baykan D. The effect of postoperative local and regional bupivacaine administered to patients with laparoscopic cholecystectomy. Van Medical Journal 2002;9(4):113-118. 
[5] Bisgaard T. Analgesic treatment after laparoscopic cholecystectomy: a critical assessment of the evidence. Anesthesiology 2006;104 (4): 835-846.

[6] Joshi GP, Kehlet H. Procedure-specific pain management: The road to improve postsurgical pain management? Anesthesiology 2013; 118: 934 - 944

[7] Dayılar H, Oyur G, Kamer E, Sarıçicek A, Cengiz F, Hacıyanlı M. Evaluation of anxiety levels of patients before colon surgery. Turk Journal of Colorectal Disease 2017;27: 6-10.

[8] Karadağ Arlı S. Evaluation of anxiety before surgery with APAIS and STAI-I scales. Hacettepe University Journal of Nursing Faculty 2017; 4(3): 38-47.

[9] Yılmaz M, Sezer H, Gürler H, Bekar M. Predictors of preoperative anxiety in surgical inpatients. Journal of Clinical Nursing 2012; (21): 956-964.

[10] Taşdemir A, Erakgun A, Deniz MN, Çertuğ A. Comparison of anxiety levels before and after surgery with State-Trait Anxiety Inventory Test in patients who were informed preoperatively. Turkish Journal of Anaesth Reanim 2013; (41) 44-49.

[11] Carr E. Barriers to effective pain management. In: Cox F, eds. Perioperative Pain Management. United Kingdom Blackwell Publishing; 2009 p.26 - 48.

[12] Koraş K, Karabulut $N$. The effect of foot massage on postoperative pain and anxiety levels in laparoscopic cholecystectomy surgery: A randomized controlled experimental study. Journal of Perianesthesia Nursing 2019; 34(3):551-558.

[13] Memedov C, Menteş Ö, Şimşek A, Kece C, Yağcı G, Harlak A. Local anesthetic infiltration to multiple sites in the prevention of postoperative pain after laparoscopic cholecystectomy: placebo-controlled comparison of ropivacaine and prilocaine. Gülhane Medical Journal 2008; 50: 84-90.

[14] Asadizaker M, Fathizadeh A, Haidari A, Goharpai S. The effect of foot and hand massage on postoperative cardiac surgery pain. International Journal of Nursing and Midwifery 2011;3 (10):165-169.

[15] Babajani S. The effect of foot reflexology massage on the level of pain during chest tuberemoval after open heart surgery. Iran Journal of Critical Care Nursing 2014 ;7(1): 15-22.

[16] Çankaya A, Sarıtaş S. Effect of classic foot massage on vital signs, pain, and nausea/vomiting symptoms after laparoscopic cholecystectomy. Surg Laparosc Endosc Percutan Tech 2018; 28(6):359- 365 .

[17] Pasyar N, Rambod MP, Kahkhaee FR. The effect of foot massage on pain Intensity and anxiety in patients having undergone a tibial shaft fracture surgery: A randomized clinical trial Journal of Orthop Trauma 2018; 32: 482-486.

[18] Ucuzal M, Kanan N. Foot massage: effectiveness on postoperative pain in breast surgery patients. Pain Management Nursing 2014;15(2): 458-465.

[19] Tsay SL, Chen NL, Chen SC, Lin HR, Lin KC. Effects of reflexotherapy on acute postoperative pain and anxiety among patients with digestive cancer. Cancer Nursing 2008; 31(2):109- 116

[20] Xue M, Fan L, Ge LN, Zhang Y, Ge JL, Gu J. Postoperative foot massage for patients after caesarean delivery. Z Geburtshilfe Neonatol 2016; 220(4):173-178.

[21] Wang HL, Keck JF. Foot and hand massage as an intervention for postoperative pain. Pain Management Nursing 2004; 5(2):59-65.
[22] Öztürk R, Sevil Ü, Sargın A,Yücebilgin MS. The effects of reflexology on anxiety and pain in patients after abdominal hysterectomy: A randomised controlled trial. Complementary Therapies in Medicine 2017; 36:107-112.

[23] Degirmen N. Effectiveness of foot and hand massage in post cesarean pain control in a group of Turkish pregnant women. Applied Nursing Research 2010; 23:153-158.

[24] Dreyer N, Cutshall SM, Huebner M, Foss DM, Lovely JK, Bauer BA. Effect of massage therapy on pain, anxiety, relaxation, and tension after colorectal surgery: a randomized study. Complementary Therapies in Clinical Practice 2015; 21:154159.

[25] Hudson BF, Davidson J, Whiteley MS. The impact of hand reflexology on pain, anxiety and satisfaction during minimally invasive surgery under local anaesthetic: A randomised controlled trial. International Journal of Nursing Studies 2015; 52: 1789-1797.

[26] Shehata AE, Elhy AA, Elsalam EAA. Effect of foot massage on pain level among patients after abdominal surgery. IOSR Journal of Nursing and Health Science 2016; 5(2): 1824.

[27] Shermeh S. Effect of foot reflex massage on sternotomy pain after coronary artery bypass graft surgery. Iranian Journal of Critical Care Nursing 2009; 2(2): 51-54.

[28] Stephenson NL, Dalton JA. Using Reflexology for Pain Management. Journal of Holistic Nursing 2003; 21(2): 179-191

[29] Akın Korhan E, Uyar M. Evidence-based approach to pain control: reflexology. Acıbadem University Journal of Health Sciences 2014; 5(1): 9-14.

[30] Aksel Wilhelm Z. Step by Step Health. Reflexology (4. edition). İstanbul Dharma Publications; 2009 p.1-57.

[31] Çevik K. Complementary and alternative therapy in nursing: reflexology. Journal of Ege University Nursing Faculty 2013; 29 (2): 71-82.

[32] Doğan D. The art of healing hands: Reflexology. Eur J Basic Med Sci 2014; 4(4): 89-94.

[33] Kandemir D, Öztekin SD. The effect of reflexology on physiological parameters and mechanical ventilation weaning time in patients undergoing open heart surgery? European Journal of Integrative Medicine 2019; 26:43 - 49.

[34] Yıldız S, Yaşa Öztürk G. Reflexology: Basic and clinical information. Journal of Integrative Medicine 2014;2(1):2642.

[35] Youssef NFA, Hassan ADA. The Effect of hand and foot massage on alleviating pain and anxiety of abdominal postoperative patients at a University Hospital: A randomized control trial. IOSR Journal of Nursing and Health Science 2017; 6 (3): 56-65.

[36] Chanif W, Petpichetchian W, Chongchareon W. Does foot massage relieve acute postoperative pain? A literature review. Nurse Media Journal of Nursing 2013; 3(1): 483 - 497.

[37] Vardanjani MM, Alavi NM, Razavi NS, Aghajani M, Azizi Fini E, Vaghefi SM. A randomized-controlled trial examining the effects of reflexology on anxiety of patients undergoing coronary angiography. Nursing and Midwifery Studies 2013; 2(3): 3-9.

[38] Abbaszadeh Y, Allahbakhshian A, Seyyedrasooli A, Sarbakhsh $P$, Goljarian S, Safaei N. Effects of foot reflexology on anxiety and physiological parameters in patients undergoing coronary artery bypass graft surgery: A clinical trial. Complementary Therapies in Clinical Practice 2018; 31: 220 - 228. 
[39] Khorsand A, Tadayonfar MA, Badiee S, Aghaee MA, Azizi $\mathrm{H}$, Baghani S. Evaluation of the effect of reflexology on pain control and analgesic consumption after appendectomy. The Journal of Alternative and Complementary Medicine 2015; 21(12): 774-780.
[40] Silverdale N, Wherry M, Roodhouse A. Massage and reflexology for post-operative cancer cystectomy patients: Evaluation of a pilot service. Complementary Therapies in Clinical Practice 2019; 34: 109-112.

How to cite this article: Abdullayev A, Ozbas A. The Effect of Foot Massage on Pain and Anxiety Levels after Laparoscopic Cholecystectomy: A Randomized-Controlled Trial. Clin Exp Health Sci 2021; 11: 746-753. DOI: 10.33808/clinexphealthsci.871454 